\title{
Hemisphere-Dependent Attentional Modulation of Human Parietal Visual Field Representations
}

\author{
Summer L. Sheremata ${ }^{1}$ and Michael A. Silver ${ }^{2}$ \\ ${ }^{1}$ Department of Psychology, George Washington University, Washington, DC 20052 and ${ }^{2}$ Helen Wills Neuroscience Institute, School of Optometry, and \\ Vision Science Graduate Group, University of California, Berkeley, California 94720
}

\begin{abstract}
Posterior parietal cortex contains several areas defined by topographically organized maps of the contralateral visual field. However, recent studies suggest that ipsilateral stimuli can elicit larger responses in the right than left hemisphere within these areas, depending on task demands. Here we determined the effects of spatial attention on the set of visual field locations (the population receptive field [pRF]) that evoked a response for each voxel in human topographic parietal cortex. A two-dimensional Gaussian was used to model the pRF in each voxel, and we measured the effects of attention on not only the center (preferred visual field location) but also the size (visual field extent) of the pRF. In both hemispheres, larger pRFs were associated with attending to the mapping stimulus compared with attending to a central fixation point. In the left hemisphere, attending to the stimulus also resulted in more peripheral preferred locations of contralateral representations, compared with attending fixation. These effects of attention on both pRF size and preferred location preserved contralateral representations in the left hemisphere. In contrast, attentional modulation of pRF size but not preferred location significantly increased representation of the ipsilateral (right) visual hemifield in right parietal cortex. Thus, attention effects in topographic parietal cortex exhibit hemispheric asymmetries similar to those seen in hemispatial neglect. Our findings suggest potential mechanisms underlying the behavioral deficits associated with this disorder.
\end{abstract}

Key words: hemispheric asymmetries; retinotopy; visual attention

\section{Introduction}

Within posterior parietal cortex, topographic mapping has been used to define areas along the intraparietal sulcus (IPS0-5) (Sereno et al., 2001; Silver et al., 2005; Schluppeck et al., 2006; Swisher et al., 2007; Silver and Kastner, 2009) that partially overlap with areas involved in visual attention (Silver et al., 2005; Szczepanski et al., 2010), visual short-term memory (Sheremata et al., 2010), and episodic memory retrieval (Hutchinson et al., 2014). These topographic maps have a contralateral bias in their representations of visual space in both hemispheres (Silver et al., 2005; Swisher et al., 2007; Szczepanski et al., 2010). In contrast, a subset of these areas exhibit task-dependent hemispheric asymmetries in visual short-term memory (IPS0-2) (Sheremata et al., 2010) and visual attention (IPS1-2) (Szczepanski et al., 2010; Szczepanski and Kastner, 2013) signals. Specifically, directing task-dependent resources toward a particular visual field location results in more bilateral representations (less contralateral bias) in the right compared with the left hemisphere.

Received June 11, 2014; revised 0ct. 11, 2014; accepted 0ct. 30, 2014.

Author contributions: S.L.S. designed research;S.L.S. performed research; S.L.S. analyzed data; S.L.S. and M.A.S. wrote the paper.

This work was supported by National Eye Institute Training Grant in Vision Science T32 EY07043, National Eye Institute Core Grant for Vision Research P30 EY003176, and National Science Foundation Major Research Instrumentation Program Grant BCS-0821855.

The authors declare no competing financial interests.

Correspondence should be addressed to Dr. Summer Sheremata, Department of Psychology, George Washington University, 2125 G Street NW, Washington, DC 20052. E-mail: ssheremata@gwu.edu.

DOI:10.1523/JNEUROSCI.2378-14.2015

Copyright $\odot 2015$ the authors $\quad 0270-6474 / 15 / 350508-10 \$ 15.00 / 0$
Although attention has been shown to modulate the locations and sizes of visual receptive fields in occipital cortex (Connor et al., 1996; Treue, 2001; Womelsdorf et al., 2006, 2008; Roberts et al., 2007; Niebergall et al., 2011), less is known about how attention affects the representation of visual space in posterior parietal cortex (but see Ben Hamed et al., 2002; Sprague and Serences, 2013). Maps of the contralateral visual field have been demonstrated in human topographic parietal cortex using fMRI, both when subjects direct attention toward the mapping stimulus and when attention is directed elsewhere (Silver et al., 2005; Swisher et al., 2007; Bressler and Silver, 2010; Bressler et al., 2013). However, standard topographic mapping methods only determine the visual field location at which a voxel is maximally activated. The population receptive field ( $\mathrm{pRF}$ ) method, in contrast, measures the fMRI response at many visual field locations, thereby determining the set of locations that evoke a response in each voxel (Dumoulin and Wandell, 2008).

We used the pRF method to test whether greater ipsilateral visual field representation in the right than left hemisphere in IPSO -5 is due to attentional modulation of the preferred locations and visual field extent of individual pRFs in parietal cortex. Subjects directed visual attention either toward or away from a stimulus that traversed the visual field. We found that, in both hemispheres, directing attention toward the stimulus resulted in larger parietal pRF sizes, corresponding to greater spatial integration across the visual field, compared with the attend fixation condition. In left IPS, attending the stimulus was also associated with $\mathrm{pRF}$ preferred locations that were further to the right (i.e., 
toward the contralateral periphery), relative to the attend fixation condition. However, there were no significant differences in pRF preferred locations between the attention conditions in right IPS. This preservation of pRF center locations, combined with larger pRF size when the mapping stimulus was attended, resulted in an increase in the proportion of the ipsilateral visual field for which stimuli evoked responses in right IPS0 -5 , compared with left IPS0 -5 .

\section{Materials and Methods}

Subjects. Nine right-handed subjects (seven female) participated in this study. All subjects provided written informed consent, and the experimental protocol was approved by the Committee for the Protection of Human Subjects at the University of California, Berkeley. One author (S.L.S.) was a subject.

Visual stimuli and experimental paradigm. Stimuli were generated using the PsychoPy software package (Peirce, 2009) and were displayed using an LCD projector (Avotec) on a screen mounted at the back of the scanner bore. Subjects viewed the stimuli via a mirror angled at $45^{\circ}$. All stimuli were presented on a mean luminance background $\left(104.8 \mathrm{~cd} / \mathrm{m}^{2}\right)$. White dots of maximum luminance $\left(209.5 \mathrm{~cd} / \mathrm{m}^{2}\right)$ were presented within a drifting bar with one of eight different orientations that swept through a circular aperture with a diameter of $28^{\circ}$ visual angle (see Fig. 1). Previous studies have demonstrated that motion within a stimulus can modulate the perceived position of that stimulus (Ramachandran and Anstis, 1990), resulting in changes in the BOLD signal within early visual cortex (Whitney et al., 2003). In our initial behavioral pilot experiments, subjects reported mislocalization of the stimulus due to dot motion within the bar. Because this illusion depends on equiluminance of the stimulus and background (Ramachandran and Anstis, 1990), we added a dark gray border $\left(25 \%\right.$ maximal luminance, $\left.52.4 \mathrm{~cd} / \mathrm{m}^{2}\right)$ along the edge of the stimulus (Fig. 1). The bar subtended a width of $5^{\circ}$ and a maximal length of $28^{\circ}$ visual angle and moved continuously across the screen at a speed of $1.375 \%$. The dots moved at a speed of $12 \%$ and had a lifetime of 40 monitor refresh frames (533 ms).

In the attend stimulus condition, subjects covertly attended the drifting bar and pressed a button whenever they detected a change in the direction of dot motion. The direction of the motion was one of four possibilities $\left(45^{\circ}, 135^{\circ}, 225^{\circ}\right.$, and $\left.315^{\circ}\right)$, and the direction of motion following each change was randomly selected. In the attend fixation condition, subjects detected a luminance decrease within a central fixation point surrounded by a square subtending $1^{\circ}$ visual angle. Intervals between changes in luminance and between changes in motion direction were always at least $800 \mathrm{~ms}$ and had a mean value of $6 \mathrm{~s}$. During each run, changes in luminance at fixation and motion coherence (percentage of dots moving in the same direction) of the stimulus were adjusted to obtain $\sim 65 \%$ performance in each task. Changes in fixation luminance and motion direction occurred in each run at random times, independently of each other, regardless of the subject's task. The duration of each sweep of the drifting bar was $24 \mathrm{~s}$. In addition, there were 4 blank intervals (12 s) in which the stimulus was not present and no changes in luminance at fixation occurred. During these intervals, subjects were instructed to simply maintain fixation.

Eight subjects completed 12 runs of pRF mapping (6 runs each of attend stimulus and attend fixation), and the remaining subject completed 3 runs of each condition. Runs were blocked in groups of 3 motion or 3 fixation runs, and the order of these blocks was counterbalanced between subjects. Subjects were instructed to continuously maintain fixation, and eye position was recorded for five subjects using an infrared camera system (Avotec) and analyzed with the ViewPoint EyeTracker software suite (Arrington Research).

Data acquisition. Data acquisition and pRF mapping were performed with a 3-Tesla Siemens MAGNETOM Trio MR scanner in the Henry H. Wheeler, Jr. Brain Imaging Center. The posterior section of a 12-channel RF head coil was used, resulting in six functional channels. Functional echo-planar images were acquired using a gradient-echo EPI sequence (repetition time $(\mathrm{TR})=2 \mathrm{~s}$; echo time $=30 \mathrm{~ms}$; voxel size $=3 \times 3 \times 2.3$ $\mathrm{mm}$, flip angle $\left.=80^{\circ}\right)$. Twenty-two slices were collected at an angle of $\sim 30^{\circ}$ from the axial plane to provide coverage of occipital and posterior parietal cortex. High-resolution whole-brain anatomical MRI images were also acquired with an intact 12-channel coil, and these were used to reconstruct the cortical surface for each subject.

Data analysis. Data were analyzed using mrVista software (http://vistalab.stanford.edu/software). Each functional time point was motion-corrected to the first acquisition of the first functional run (Jenkinson et al., 2002). The first six time points were removed from each run, and pRFs were estimated in native functional space for each subject using the method described by Dumoulin and Wandell (2008). The data were then aligned to each subject's high-resolution anatomical scan using a T1-weighted image that was coplanar with the EPI images and were projected onto each subject's cortical surface. pRF centers were converted into polar spatial coordinates to define topographic regions in occipital and parietal cortices.

The circular stimulus aperture (radius of $14^{\circ}$ ) necessitated selection of voxels with pRFs centered at $<10^{\circ}$ eccentricity, as it was not possible to accurately model the entire extent of more peripheral pRFs. In addition, the bar-shaped stimulus ( $5^{\circ}$ width) that swept across the stimulus aperture in multiple directions precluded scaling of stimulus size as a function of visual field eccentricity. This imposes limitations on the estimation of small pRFs at parafoveal and foveal locations in early visual cortex.

Only pRFs with response coherence $>0.18$ were included in the analysis. This choice of coherence threshold was based on a tradeoff between maximizing the number of analyzed voxels while excluding voxels with a poor model fit. This yielded an average of $80 \%$ of the voxels within the set of topographic ROIs and a minimum of 100 voxels per subject in each of the topographic parietal areas (IPS0-5), thereby minimizing the possibility that spurious differences between attention conditions occurred due to sampling a very small subset of voxels in a given area.

Estimates of pRF size $(\sigma)$ and center $\left(\mathrm{x}_{0}, \mathrm{y}_{0}\right)$ were derived using the pRF mapping functions in the mrVista imaging software package (Dumoulin and Wandell, 2008). Based on these parameters, a lateralization index was defined as follows:

$$
\frac{1}{\sqrt{\pi}} \int_{\frac{x_{0}}{\sqrt{2} \sigma}}^{+\infty} e^{-t^{2}} d t
$$

This index is the integral of a normalized Gaussian function, where $e^{-t^{2}}$ is the pRF (Gaussian function), $\mathrm{x}_{0}$ is the pRF center location, and $\sigma$ is the size of the pRF (the SD of the Gaussian fit, in degrees of visual angle). Because these lateralization index values are bounded between 0 and 1 , Fisher's $z$-transformation was applied to all lateralization index values before statistical analysis.

To compare lateralization index values between the hemispheres, we subtracted the lateralization index in the left hemisphere from 1 . This resulted in lateralization index values for each voxel that had a maximum of 1 in the contralateral periphery, 0.5 at the vertical meridian, and a minimum of 0 in the ipsilateral periphery.

\section{Results}

We recorded BOLD responses in individual voxels as subjects viewed a bar composed of moving dots that traversed the visual field. In separate runs, subjects performed either a luminance change detection task at fixation (attend fixation) or a motion direction change detection task within the mapping stimulus (attend stimulus) (Fig. 1). There was no significant difference in task difficulty between the two attention conditions, as assessed by the percentages of events that were correctly detected (attend fixation: $68.7 \pm 5.8 \%$; attend stimulus: $64.6 \pm 4.7 \%$; $t_{(8)}=$ $0.52, p=0.61)$.

pRFs were estimated by comparing the time series from each voxel to the time series resulting from convolution of the stimulus sequence with a 2-D Gaussian centered at different visual field locations (Dumoulin and Wandell, 2008). Values for the center $\left(\mathrm{x}_{0}, \mathrm{y}_{0}\right)$ and the size $(\sigma)$ of the pRF for each voxel were deter- 

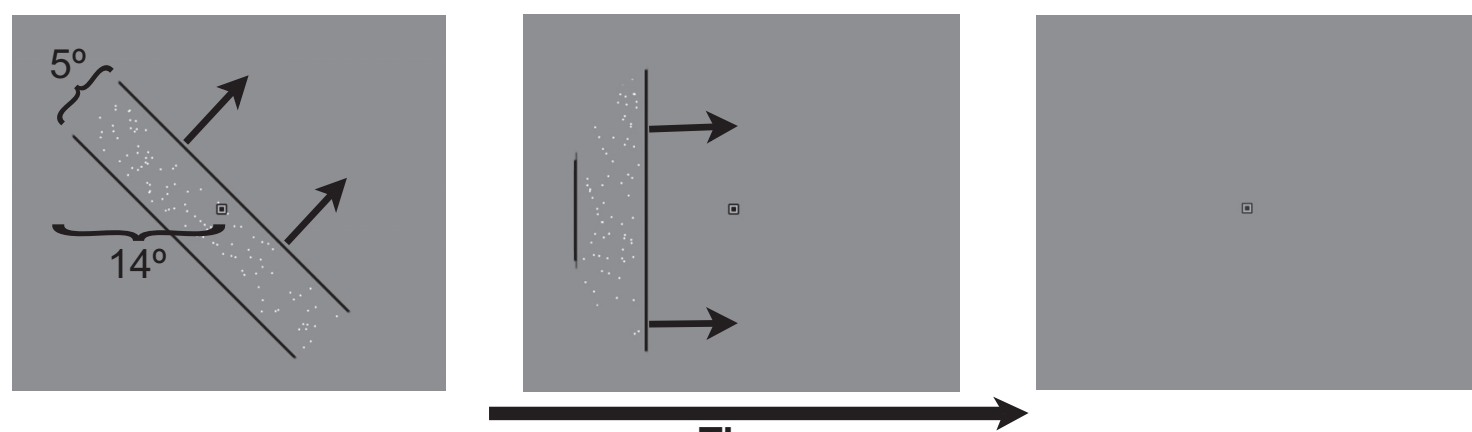

Time

Figure 1. Stimuli used for pRF mapping. Coherent dot motion was presented within a drifting bar (4 orientations, 8 directions of drift) that included a dark gray border. The bar had a width of $5^{\circ}$ and was swept through a circular visual aperture with a radius of $14^{\circ}$. The dimensions of this aperture determined the length of the bar at any given time. In addition, four periods with no visual stimulation were presented to estimate baseline activity.

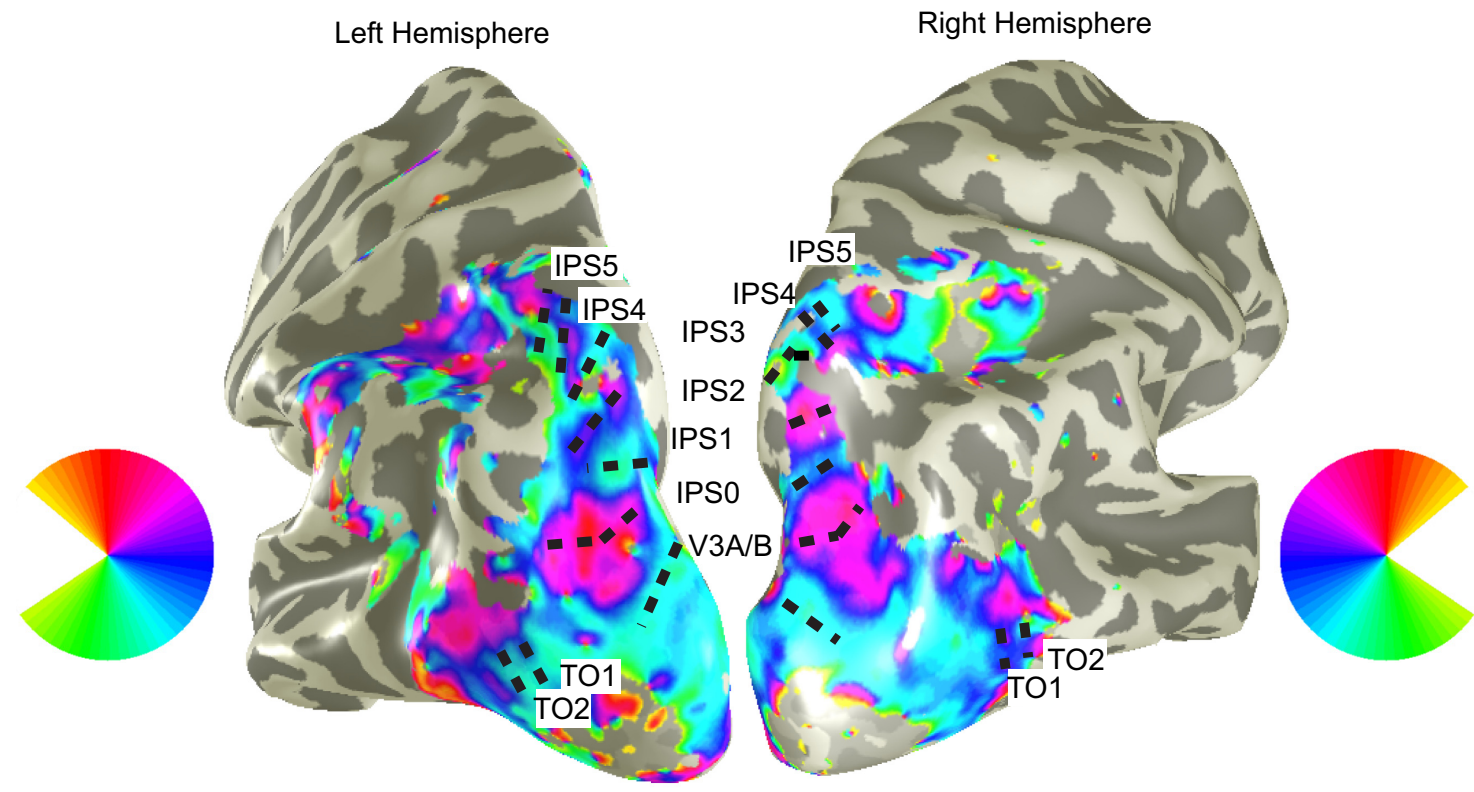

Figure 2. Maps of pRF center locations (displayed in polar visual field coordinates) for an individual subject. The color wheels indicate the location in the contralateral visual field corresponding to each location on the cortex for the left and right hemispheres.

mined that minimized the mean squared error between the predicted and observed fMRI time series.

To define the locations and boundaries of topographic areas, time series were averaged across both attention conditions. Across all subjects, areas IPS0 -5 were defined in each hemisphere based on the spatial distribution of pRF centers on the cortical surface (Fig. 2). The degree of contralateral bias of the visual field representation was estimated for each voxel using a lateralization index with values ranging from 0 (completely ipsilateral representation) to 1 (completely contralateral representation) (see Materials and Methods).

To assess the effects of spatial attention on lateralization of visual representations, we performed a within-subject ANOVA with factors of attention condition, hemisphere, and ROI (IPSO$5)$. There was a significant main effect of $\operatorname{ROI}\left(F_{(5,40)}=3.42, p<\right.$ $0.05)$, reflecting less lateralization in more anterior portions of IPS (Fig. 3). After controlling for multiple comparisons using the false discovery rate, paired $t$ tests of all pairwise combinations of IPS areas (collapsed across hemispheres) demonstrated greater lateralization in IPS0 than IPS5 $\left(t_{(8)}=6.78, p<0.01\right)$ and in IPS1 compared with IPS3 $\left(t_{(8)}=4.31, p<0.05\right)$ and IPS5 $\left(t_{(8)}=5.52\right.$, $p<0.01)$. There was also a significant interaction between ROI and attention condition $\left(F_{(5,40)}=4.85, p<0.01\right)$. Post hoc testing in each IPS area revealed reduced lateralization for the attend stimulus compared with the attend fixation condition in IPS2 $\left(t_{(8)}=4.09, p<0.01\right)$.

Importantly, lateralization index values exhibited a significant interaction between attention condition and hemisphere $\left(F_{(1,8)}=5.45, p<0.05\right)$ (Fig. 3). Because there was no significant interaction between hemisphere and ROI, we combined all six parietal areas to create an inclusive IPS ROI in each hemisphere (IPS0 -5). For this inclusive ROI, an ANOVA with factors of hemisphere and attention condition showed a significant interaction $\left(F_{(1,8)}=6.68, p<0.05\right)$. Specifically, the lateralization index was significantly lower in the attend stimulus condition than the attend fixation condition in the right hemisphere $\left(t_{(8)}=\right.$ $3.12, p<0.05)$. In contrast, there was no significant difference between attention conditions in the left hemisphere $t_{(8)}=1.62$, $p=0.14)$.

Moreover, for the attend stimulus condition, lateralization index values were significantly lower in the right than left hemi- 

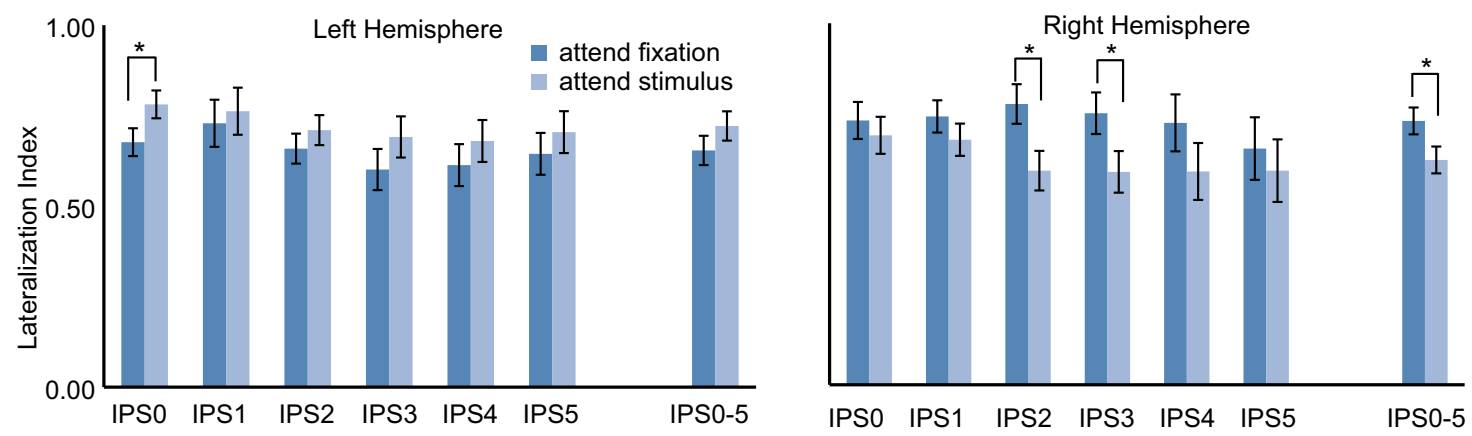

Figure 3. Mean lateralization index values for individual IPS areas (IPS0-IPS5) as well as the combined parietal ROI (IPSO - 5). Error bars indicate the SE of the difference of the mean lateralization index values. Across ROIs, attending to the stimulus significantly decreased lateralization of the visual field representation in right but not left IPS.

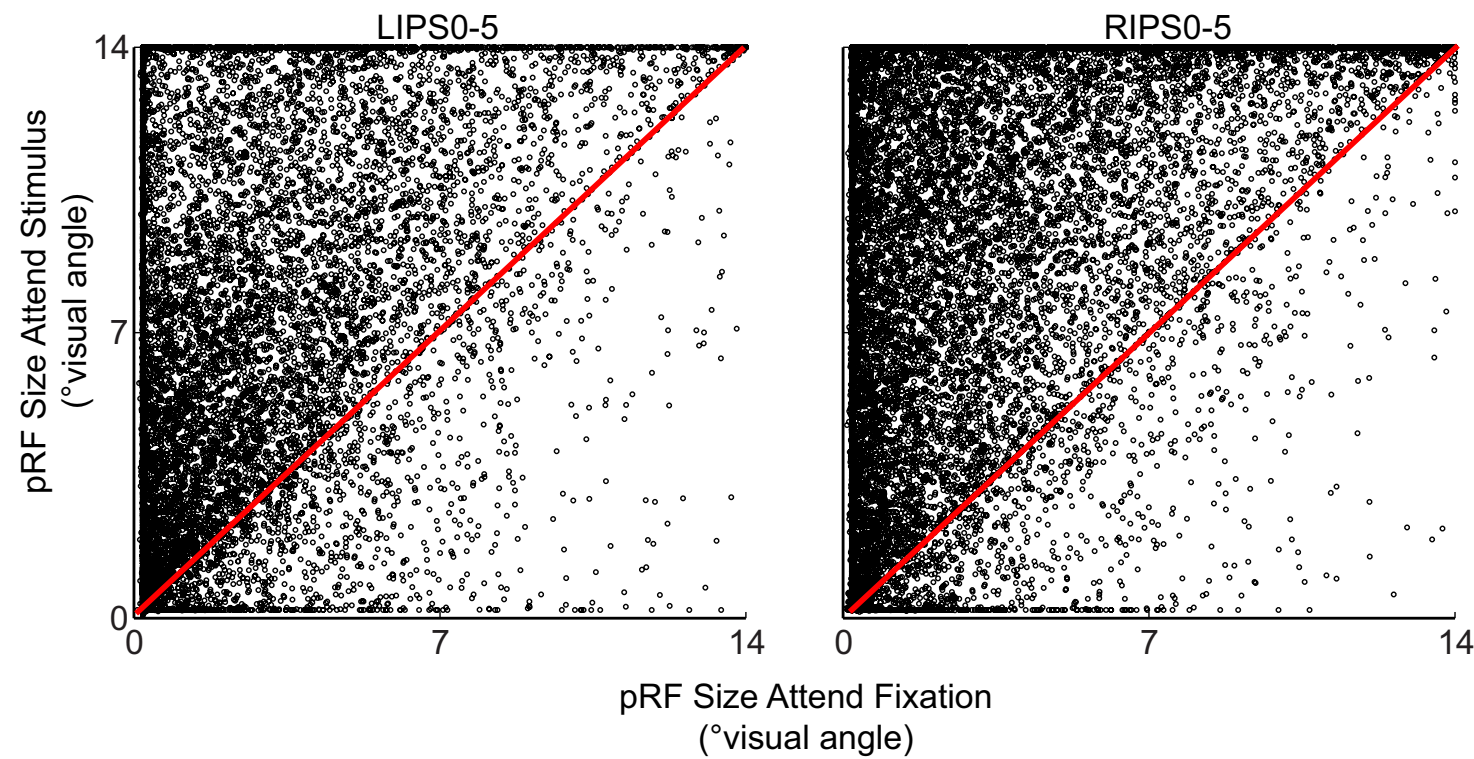

Figure 4. Plots of pRF size values for individual voxels for attend fixation and attend stimulus conditions for the combined ROI (IPSO - 5) in the left and right hemispheres. Most voxels fall above the red unity line, demonstrating greater overall pRF size in the attend stimulus relative to the attend fixation condition.

sphere $\left(t_{(8)}=2.94, p<0.05\right)$. However, the attend fixation condition showed no significant difference between the hemispheres $\left(t_{(8)}=1.10, p=0.30\right)$. These results suggest that allocating attention toward the mapping stimulus resulted in an asymmetrical representation of the visual field across the two hemispheres.

The lateralization index is estimated from two parameters: the size and the horizontal center location of the pRF. To determine how attention differentially affected these two measures, we performed separate within-subject ANOVAs for $\mathrm{pRF}$ size and center location for the inclusive IPS0-5 ROIs. There was a significant main effect of attention condition on pRF size $\left(F_{(1,8)}=116.35\right.$, $p<0.001)$ but no interaction between hemisphere and attention condition $\left(F_{(1,8)}=0.01, p=0.98\right)$. Post hoc testing demonstrated significantly larger pRFs when attending to the stimulus compared with attending fixation in both hemispheres (left: $t_{(8)}=$ 7.72, $p<0.001$; right: $t_{(8)}=10.1, p<0.001$ ) (Fig. 4). Specifically, across both hemispheres, mean pRF size was $6^{\circ}$ when directing attention to the stimulus but only $3^{\circ}$ when attention was directed to fixation.

One possible explanation of this attentional modulation of pRF size is that attending the stimulus resulted in greater overall response amplitude, compared with attending the fixation point. This would potentially cause an "iceberg effect," in which greater response reliability could cause the flanks of the pRF to exhibit significant responses only in the attend stimulus condition, thereby yielding larger estimates of pRF size. To test this possibility, we measured the correlation between the effects of attention on pRF size and on amplitude for each voxel. Across subjects, there was no significant correlation between these attention effects (left IPS: mean $r=-0.04$, range -0.13 to 0.04 ; right IPS: mean $r=-0.04$, range, -0.30 to 0.16 ). We conclude that larger $\mathrm{pRF}$ size in the attend stimulus relative to the attend fixation condition in parietal cortex is not due to attention effects on response amplitude. This is consistent with a recent report that also found independence of attentional modulation of pRF size and pRF amplitude (Sprague and Serences, 2013).

In contrast to the symmetric effects of attention on pRF size in the two hemispheres, we observed hemispheric asymmetries in the effects of attention on pRF center locations. In the attend fixation condition, the visual field representation is strongly biased toward the contralateral visual field in both hemispheres (Fig. 5). In the absence of attentional modulation of pRF preferred location, however, larger $\mathrm{pRF}$ sizes in the attend stimulus than the attend fixation condition would lead to a less lateralized (more bilateral) visual field representation, as we observed in the right hemisphere (Fig. 5). However, the left hemisphere had equivalent lateralization of visual field representation in both attention conditions (Fig. 5), consistent with larger pRF sizes as 

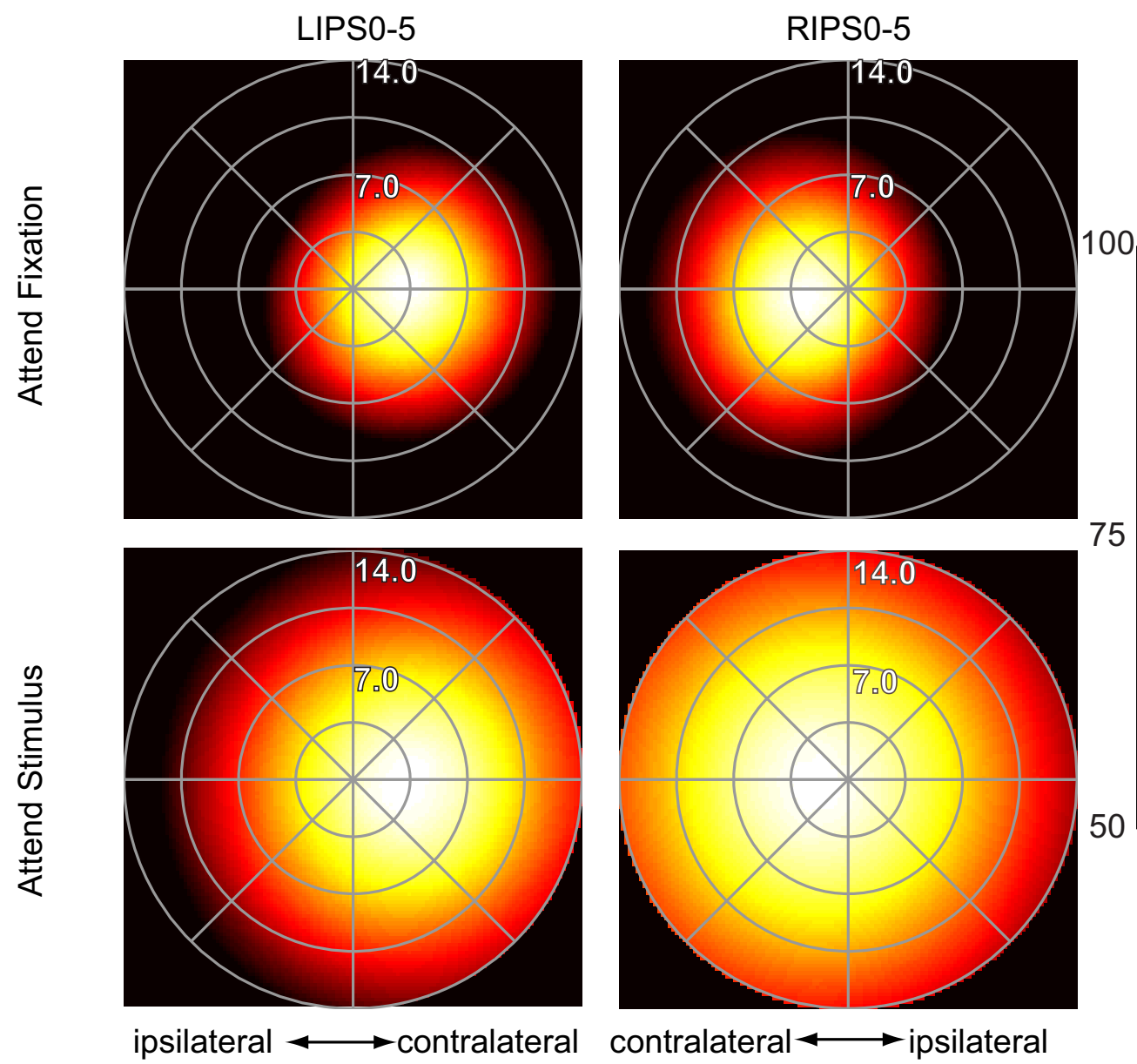

75

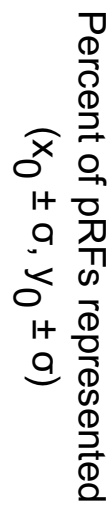

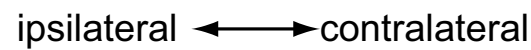

contralateral

\section{visual field location}

Figure 5. Attentional modulation of visual field representations in the combined IPS ROIs (IPSO - -5). pRF center locations and extent $\left(x_{0} \pm \sigma, y_{0} \pm \sigma\right)$ of each voxel were projected onto the visual field for each subject. Each figure panel represents the percentage of voxels whose pRF overlapped each visual field location. In the attend fixation condition, visual field representations were mainly confined to the contralateral visual hemifield. However, in the attend stimulus condition, visual field representations extended substantially into the ipsilateral visual hemifield in the right, but not left, hemisphere.

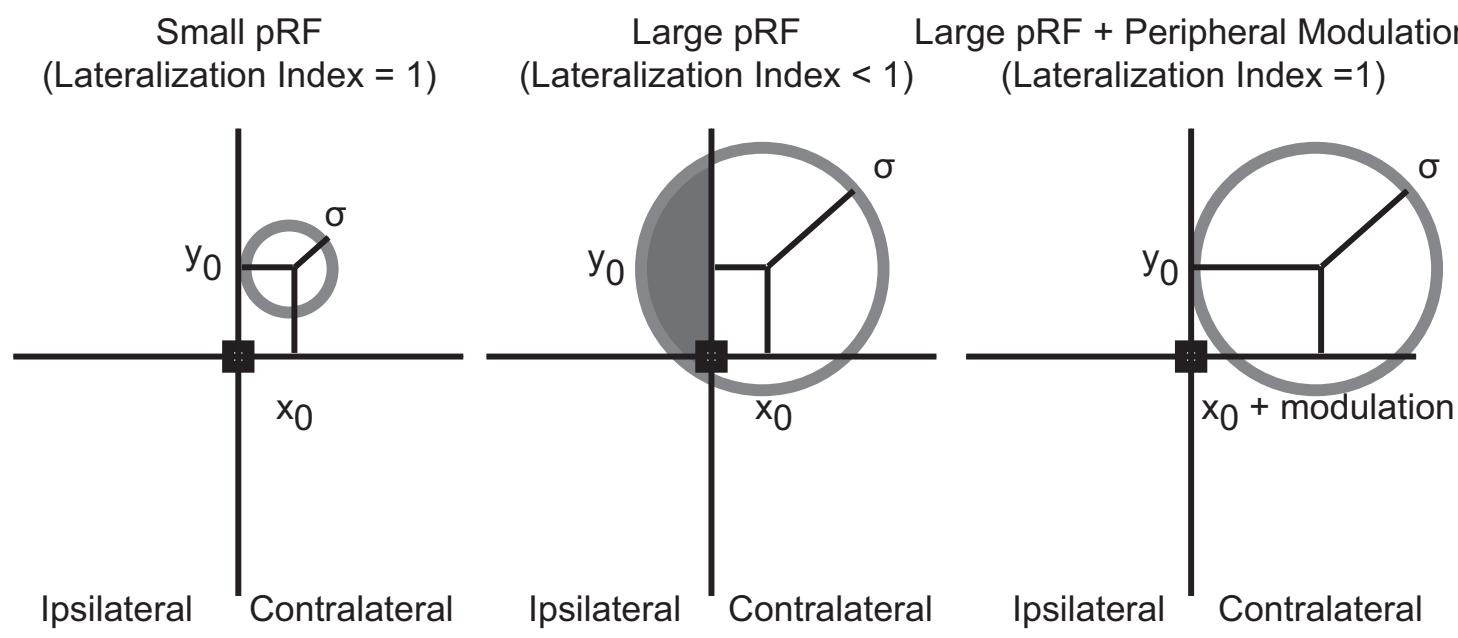

Figure 6. Schematic diagrams of different effects of attention on pRF size $(\sigma)$ and horizontal center position $\left(\mathrm{x}_{0}\right)$. Left, In the attend fixation condition, the small pRF is completely within the contralateral visual hemifield, resulting in a lateralization index of 1 . Middle, In the right hemisphere, greater pRF size with no change in pRF position in the attend stimulus condition results in expansion of the PRF into the ipsilateral visual hemifield (shaded in gray), reducing the lateralization index to $<1$. Right, In the left hemisphere, greater pRF size combined with a more contralateral peripheral location of pRF centers in the attend stimulus condition results in a larger pRF that is still within the contralateral visual field, maintaining a lateralization index of 1. 
A

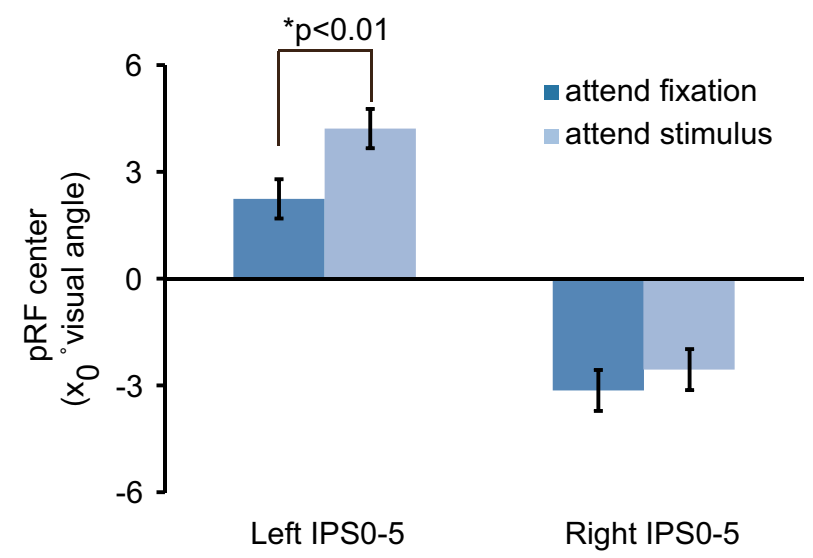

B

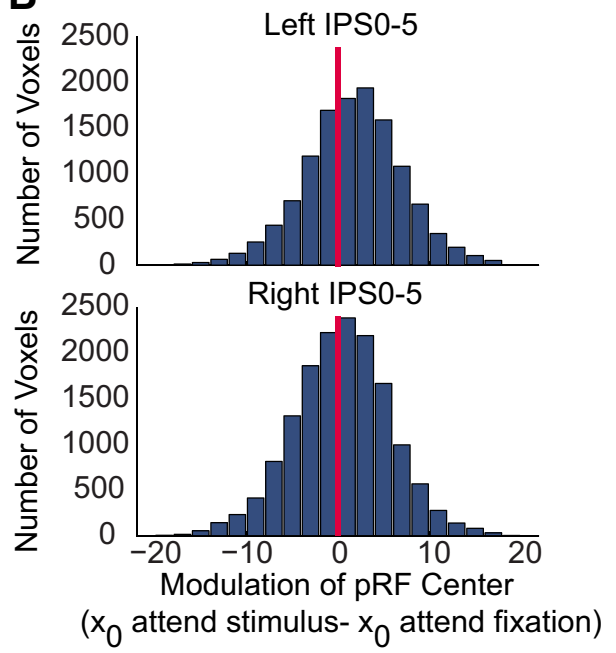

Figure 7. A, pRF horizontal center locations for attend fixation and attend stimulus conditions in IPSO - 5. Error bars indicate the SE of the difference of the means. In the left hemisphere, pRF preferred location was significantly more peripheral in the contralateral visual field when subjects attended the stimulus versus when they attended the fixation point. In contrast, there was no attentional modulation of pRF preferred location in the right hemisphere. $\boldsymbol{B}$, Histograms of attentional modulation of the preferred location of $\mathrm{pRF}$ of individual voxels in IPSO -5 , collapsed across subjects. Red lines indicate no change in pRF center location across attention conditions.

well as more contralateral peripheral pRF preferred locations in the attend stimulus condition relative to the attend fixation condition (Fig. 6).

Indeed, an ANOVA of pRF center location (absolute value of $\mathrm{x}_{0}$ ) revealed a significant main effect of attention condition $\left(F_{(1,8)}=5.91, p<0.05\right)$ as well as an interaction between hemisphere and condition $\left(F_{(1,8)}=6.94, p<0.05\right)$ (Fig. 7). In the left hemisphere, $\mathrm{pRF}$ locations were significantly further toward the right visual field (more contralateral) in the attend stimulus compared with the attend fixation condition $\left(t_{(8)}=3.58, p<0.01\right)$. In contrast, pRF center locations in the right hemisphere did not significantly differ between the attention conditions $\left(t_{(8)}=1.02\right.$, $p=0.34$ ). Furthermore, in the attend stimulus condition, $\mathrm{pRF}$ locations were located more peripherally in the left hemisphere than the right hemisphere $\left(t_{(8)}=2.92, p<0.05\right)$, but there was no significant difference in absolute $\mathrm{x}_{0}$ values between the hemispheres for the attend fixation condition $\left(t_{(8)}=1.12, p=0.29\right)$.

These hemispheric asymmetries in lateralization could reflect differences in task performance across the visual field. Specifically, if detection of changes in stimulus motion was more difficult in the left than right visual hemifield, this could account for hemispheric differences in attentional modulation of pRF preferred locations. We tested this by comparing motion direction discrimination performance for stimulus locations in the left and right periphery, excluding the $2 \mathrm{~s}$ interval during which the bar traversed the vertical meridian. There was no significant effect of side of stimulus location on behavioral performance in the attend stimulus condition (left: mean $62.5 \pm 6.0 \%$; right: $62.1 \pm 5.4 \%$; $\left.t_{(8)}=0.07, p=0.94\right)$, suggesting that differences in performance in the two visual hemifields are not likely to account for the differential effects of attention in the two hemispheres that we have observed.

\section{Hemispheric asymmetries in effects of spatial attention in} parietal cortex do not generalize to occipital cortex

Using a similar stimulus and task to those we have used, a recent study reported that neuronal receptive field size in macaque MT was larger during covert tracking of a moving stimulus compared with attending to central fixation (Niebergall et al., 2011). We topographically defined motion-responsive temporal occipital cortical areas (TO1 and TO2; thought to be homologous to the macaque $\mathrm{MT}^{+}$complex) in both hemispheres of all of our subjects (Amano et al., 2009). Consistent with results from single neurons in macaque MT (Niebergall et al., 2011), we found that pRF size in a combined TO1/2 ROI was larger when subjects attended the stimulus, relative to attending the fixation point $\left(F_{(1,8)}=28.30, p<0.001\right)$.

In contrast to IPS -5 , we found a significant main effect of attention on lateralization index values in TO1/2 $\left(F_{(1,8)}=5.26\right.$, $p=0.05$ ) that was due to significantly larger lateralization index values (i.e., greater contralateral representation) in the attend stimulus condition across hemispheres $\left(t_{(8)}=2.29, p=0.05\right)$. Furthermore, there was no significant interaction between attention condition and hemisphere for lateralization index values in $\mathrm{TO} 1 / 2\left(F_{(1,8)}=2.89, p=0.13\right)$. For pRF preferred location, there was a main effect of attention condition $\left(F_{(1,8)}=34.03, p<\right.$ 0.001) (Fig. 8). That is, in both hemispheres, larger TO1/2 pRF sizes in the attend stimulus condition were offset by more peripheral preferred pRF locations, thereby preserving the contralateral bias of the visual field representations in these areas. There was no significant interaction between attention condition and hemisphere for either pRF size $\left(F_{(1,8)}=0.023, p=0.88\right)$ or preferred location $\left(F_{(1,8)}=0.08, p=0.79\right)$.

Finally, we tested whether hemispheric asymmetries in attention effects were present in early visual cortex. There was no significant main effect of attention condition on lateralization index values in retinotopically defined early visual areas V1-V3 $(F=2.32, p=0.17)$. For pRF size, a significant effect of ROI $\left(F_{(2,16)}=12.37, p<0.001\right)$ reflected increases in $\mathrm{pRF}$ size from $\mathrm{V} 1$ to $\mathrm{V} 3\left(\mathrm{~V} 1<\mathrm{V} 2: t_{(8)}=2.85, p<0.05 ; \mathrm{V} 1<\mathrm{V} 3: t_{(8)}=3.55, p<\right.$ $\left.0.01 ; \mathrm{V} 2<\mathrm{V} 3: t_{(8)}=3.86, p<0.01\right)$. There was also a significant interaction between attention condition and ROI $(F=5.72, p<$ $0.05)$. Specifically, compared with attending fixation, attending the stimulus was associated with larger pRF sizes in V2 $t_{(8)}=$ 2.43, $p<0.05)$ and V3 $\left(t_{(8)}=2.26, p=0.05\right)$ but not V1 $\left(t_{(8)}=\right.$ 1.21, $p=0.26)$.

Consistent with areas TO1/2 and left IPS, there was also a main effect of attention on pRF center locations in early visual 


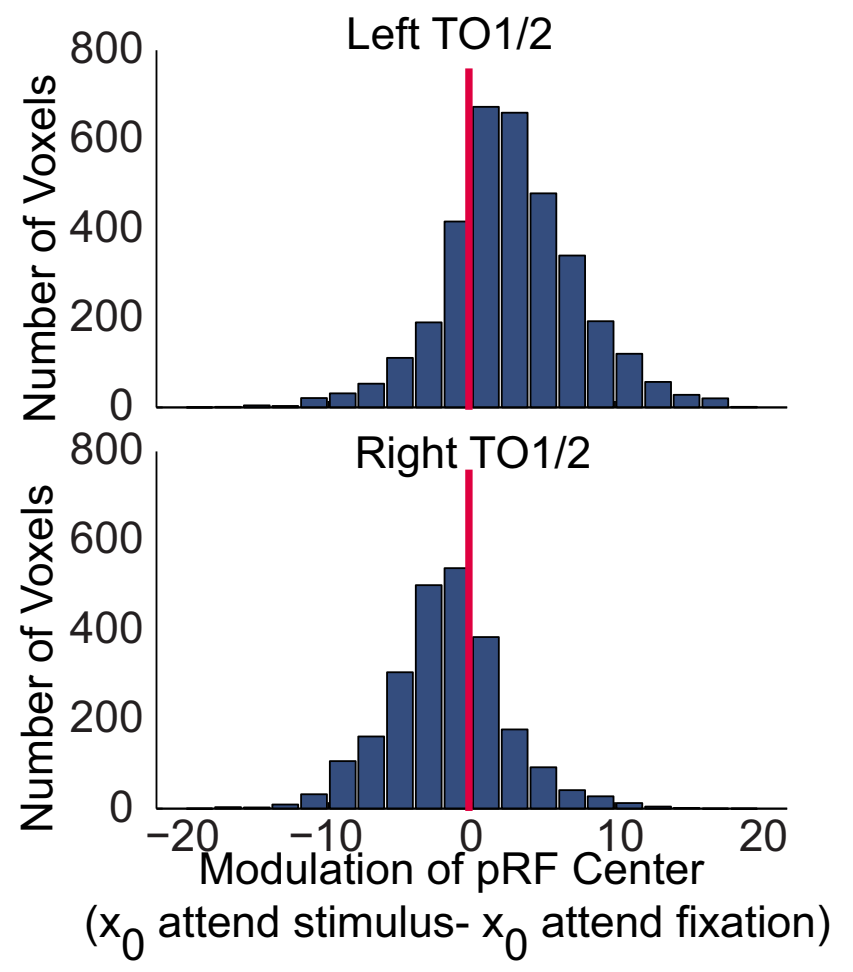

Figure 8. pRF center locations were more peripheral in the contralateral hemisphere in the attend stimulus versus attend fixation condition in T01/2. In contrast to IPS areas, this attentional modulation of pRF preferred location occurred in both left and right hemispheres in T01/2. Red lines indicate no change in PRF center location across attention conditions.

cortex $\left(F_{(1,8)}=8.36, p<0.05\right)$ because of more peripheral preferred locations of pRFs in the attend stimulus compared with attend fixation condition $\left(t_{(8)}=2.89, p<0.05\right)$. Importantly, there were no significant interactions in early visual cortex between hemisphere and attention condition for lateralization index, pRF size, or pRF center location. Together, the results from visual cortex demonstrate that the hemispheric asymmetries in the effects of spatial attention on parietal visual field representations do not generalize to occipital cortex.

\section{Eccentricity dependence of attentional modulation of pRF size and preferred location}

Attentional modulation has been shown to be eccentricity dependent in multiple topographically organized occipital and parietal cortical areas (Roberts et al., 2007; Bressler et al., 2013). We therefore examined the eccentricity dependence of attentional modulation of pRF size and preferred visual field location. All voxels having pRFs with preferred locations in the contralateral hemifield (calculated from data combined from the two attention conditions) were binned based on eccentricity of their preferred locations. To examine the effect of eccentricity on attentional modulation of pRF size and preferred location, a linear function was fit to plots of attentional modulation (difference in either pRF size or location in the two attention conditions) versus eccentricity.

For pRF size in IPS0-5, the slopes of the linear fits were not significantly different from zero in either hemisphere, indicating no reliable eccentricity dependence in the effects of attention on pRF size. In contrast, for pRF preferred location, the slopes of the linear fits were significantly less than zero in left but not right IPS0-5 (left IPS0-5: slope $=-0.23, \mathrm{CI}=0.23$; right IPS0 -5 : slope $=0.12, \mathrm{CI}=0.24)$, demonstrating that attentional modulation of pRF center position occurred in an eccentricitydependent manner in left, but not right, IPSO $-5\left(t_{(8)}=2.30\right.$, $p=0.05)$. The negative slope in left IPS0 -5 indicates that $\mathrm{pRFs}$ located closest to the vertical meridian showed the largest effects of attention on pRF preferred location.

Area TO1/2 exhibited a small but significantly positive slope in the plot of eccentricity dependence of attentional modulation of pRF size in both hemispheres (left TO1/2: slope $=0.22, \mathrm{CI}=$ 0.17 ; right $\mathrm{TO} 1 / 2$ : slope $=0.25, \mathrm{CI}=0.21$ ) that was driven by relatively small increases in $\mathrm{pRF}$ size near the vertical meridian. In contrast to IPS0 -5 , the effects of eccentricity on attentional modulation of pRF preferred location in TO1/2 showed no evidence for a difference between the left and right hemispheres: the slopes of the linear fits were not significantly different than zero in either hemisphere, consistent with pRF preferred locations being more peripheral (in the contralateral visual field) in the attend to stimulus condition, independent of eccentricity. Importantly, there were no significant differences between left and right TO1/2 in slopes for attentional modulation of either pRF size or location.

Finally, we measured the eccentricity dependence of attentional modulation of pRF size and center location in early visual cortex. Because our previous analyses revealed some differences across V1-V3, we performed an ANOVA on slope of the linear fit, with main effects of hemisphere and ROI. For attentional modulation of pRF size, a significant main effect of ROI $\left(F_{(2,8)}=4.06\right.$, $p<0.05$ ) reflected a relatively lower slope value in $\mathrm{V} 1$, although none of the slope values was significantly different from zero in $\mathrm{V} 1, \mathrm{~V} 2$, or V3. There were also no significant main effects of hemisphere or interaction between ROI and hemisphere for $\mathrm{pRF}$ size. For attentional modulation of $\mathrm{pRF}$ location, there were no significant main effects of either ROI or hemisphere on slope values and no significant interaction between ROI and hemisphere. Overall, our analyses reveal no evidence for a reliable eccentricity dependence of attentional modulation of pRF size or preferred location in early visual cortex.

\section{Attentional modulation of vertical locations of $\mathrm{pRF}$ centers}

The effects of spatial attention on pRF preferred locations along the horizontal axis ( $\mathrm{x}_{0}$ parameter of the Gaussian model) that we describe above could be specific to the horizontal direction or could reflect more general attentional modulation of eccentricity of pRF preferred locations. To distinguish between these possibilities, we compared pRF preferred location along the vertical axis ( $y_{0}$ parameter) for the attend stimulus and attend fixation conditions. Voxels were classified as upper or lower visual field based on the location of their pRF center in data combined from both attention conditions, and ANOVAs were conducted with factors of visual field (upper versus lower), hemisphere, and attention condition. To compare upper and lower visual fields, absolute values of $\mathrm{y}_{0}$ (i.e., distance from the horizontal meridian) were used.

Importantly, no main effect of attention or interaction with attention was found in any of the areas. For IPS0 -5, TO1/2, and $\mathrm{V} 1-\mathrm{V} 3$, tests for a main effect of attention condition, an interaction between attention condition and visual field, an interaction between attention condition and hemisphere, and a three-way interaction (attention condition by visual field by hemisphere) were all nonsignificant.

In contrast, visual field and hemisphere biases existed independent of attention condition. Across all areas, a main effect of visual field (IPS0 -5: $F_{(1,8)}=7.43, p<0.05$; TO1/2: $F_{(1,8)}=16.03$, $\left.p<0.01 ; \mathrm{V} 1-\mathrm{V} 3: F_{(1,8)}=22.60, p<0.01\right)$ reflected more periph- 
eral visual field representations in the lower visual field compared with the upper visual field (IPS0 $-5: t_{(8)}=2.73, p<0.05$; TO1/2: $t_{(8)}=4.00, p<0.01$; V1-V3: $\left.t_{(8)}=4.55, p<0.01\right)$. These anisotropies are consistent with greater representation of the lower visual field reported in nonhuman primate striate (Van Essen et al., 1984; Tootell et al., 1988) and middle temporal cortex (Maunsell and Van Essen, 1987) as well as in human lateral (Sayres and Grill-Spector, 2008) and temporal occipital cortex (Amano et al., 2009).

Main effects of hemisphere were also evident but were not consistent across cortical areas. In IPS0 -5, a main effect of hemisphere $\left(F_{(1,8)}=13.19, p<0.01\right)$ reflected more peripheral representations in right compared with left IPS -5 across attention conditions (lower visual field representation in left IPS0 -5: mean $\mathrm{y}_{0}=-3.92$; lower visual field representation in right IPS0-5: mean $\mathrm{y}_{0}=-4.67$; upper visual field representation in left IPS0 -5: mean $\mathrm{y}_{0}=3.00$; upper visual field representation in right IPS0-5: mean $\mathrm{y}_{0}=3.42$ ). In V1-V3, a main effect of hemisphere $\left(F_{(1,8)}=12.02, p<0.01\right)$ reflected more peripheral representations in the left than right hemisphere (lower visual field representation in left V1-V3: mean $\mathrm{y}_{0}=-4.81$; lower visual field representation in right V1-V3: mean $\mathrm{y}_{0}=-4.17$; upper visual field representation in left V1-V3: mean $\mathrm{y}_{0}=3.79$; upper visual field representation in right V1-V3: mean $\left.y_{0}=2.92\right)$. No significant main effect of hemisphere in TO1/2 was found, and there were no significant interactions between visual field and hemisphere in IPS0 -5, T01/2, or V1-V3.

These results differ from those from the analysis of attentional modulation of horizontal pRF preferred location in several respects. First, there was clear evidence of hemispheric differences in attentional modulation in IPS0 -5 for horizontal location but not for vertical location. Second, horizontal pRF preferred locations were at greater eccentricity (more in the contralateral periphery) in the attend stimulus compared with the attend fixation condition in both left and right TO1/2 and early visual cortex, but we observed no significant attentional modulation of vertical position in these areas. These findings provide evidence that the attentional modulation of horizontal pRF location does not reflect a general eccentricity effect.

\section{Eye tracking analyses}

An apparent hemispheric asymmetry in attentional modulation of visual field representations could arise from effects of attention on patterns of eye position or eye movements. Across subjects, we found no significant difference between attention conditions in mean horizontal eye position (attend fixation: mean $=0.05^{\circ}$, $\mathrm{CI}=\left[-2.02^{\circ}, 2.70^{\circ}\right]$; attend stimulus: mean $=0.07^{\circ}, \mathrm{CI}=$ $\left.\left[-0.94^{\circ}, 1.23^{\circ}\right] ; t_{(4)}=0.65, p=0.55\right)$. Specifically, to account for the observed attentional modulation of lateralization of visual field representations in parietal cortex in the right, but not left, hemisphere, eye position would have to be systematically biased toward the left in the attend stimulus condition compared with the attend fixation condition. This systematic shift in one direction, but not the other, would have resulted in a distribution of eye positions that was skewed with respect to left versus right visual field. We therefore compared the skewness of eye position distributions for each subject for the two attention conditions and found no significant difference $\left(t_{(4)}=0.75, p=0.49\right)$.

If there were greater variability in eye position in the attend stimulus compared to the attend fixation condition, this could lead to apparent attentional modulation of pRF size and/or location. Specifically, attending to the stimulus could result in a larger spread of gaze position (which would increase pRF size) and/or gaze positions that were biased toward the location of the mapping stimulus (which could increase eccentricity of pRF preferred locations). We therefore measured the SD in eye position for each attention condition. Of the 5 subjects for which we had eyetracking data in the scanner, two had greater SDs in the attend fixation condition and three had greater SDs in the attend stimulus condition. On average, the SD of eye position was 1.0 degrees for the attend fixation condition and 1.3 degrees for attend stimulus condition $\left(t_{(4)}=1.52, p=0.19\right)$. We therefore conclude that the hemispheric differences in the effects of attention on parietal visual field representations are not due to effects of eye position.

\section{Discussion}

We have demonstrated a remarkable attentional modulation of visual field representations in topographic posterior parietal cortex that is fundamentally different in the two hemispheres. For voxels in IPS0 -5, TO1/2, V2, and V3 in both left and right hemispheres, the portion of the visual field for which a stimulus evoked a visual response was greater when subjects directed attention to the stimulus compared with a central fixation point. For some voxels, larger pRF sizes in the attend stimulus condition resulted in pRFs that crossed the vertical meridian, yielding greater representation of ipsilateral visual field locations within that voxel. Indeed, this was observed for many voxels in topographic IPS in the right hemisphere. In contrast, in left IPS (and in TO1/2 and early visual cortex in both hemispheres), contralateral preference of the visual field representation was similar for the two attention conditions because larger pRF sizes in the attend stimulus condition were offset by more peripheral pRF preferred locations in the contralateral visual field.

Our results provide an explanation of apparent discrepancies in the visual field mapping and visual attention and short-term memory literatures. Specifically, across both hemispheres, regions of IPS have been defined based on their topographic representations of locations in the contralateral visual field. However, recent studies have demonstrated that responses in a subset of these regions exhibit less contralateral bias in the right, compared with the left, hemisphere (Sheremata et al., 2010; Szczepanski et al., 2010; Szczepanski and Kastner, 2013). In traditional retinotopic mapping studies that use stimuli that periodically traverse the visual field (such as rotating wedges and expanding/contracting rings), the location within the visual field that elicits the greatest response is computed for each voxel. This location is equivalent to the center of the pRF. In our data, $\mathrm{pRF}$ center locations were mainly in the contralateral visual field in topographically organized areas in both hemispheres, regardless of attention condition. However, analysis of the effects of attention on both pRF size and center location demonstrated that attending to the stimulus was associated with enhanced ipsilateral visual field representations in right parietal cortex.

In our experiments, we directly compared conditions in which attention was directed toward the stimulus versus toward the fixation point. Given that this approach does not include a pure baseline attention condition, we cannot independently measure the contributions of the attend stimulus and attend fixation conditions to our results. However, a direct comparison of the two hemispheres revealed significant differences in lateralization index values and pRF preferred locations in the attend stimulus but not in the attend fixation condition. These findings provide evidence that the hemispheric asymmetries in attentional modulation we report are driven by the attend stimulus condition.

The pRF model we have used (Dumoulin and Wandell, 2008) fits a circular Gaussian to the responses of every voxel and incor- 
porates all tested visual field locations. However, evidence from single-unit recordings suggests that spatial attention can draw neuronal receptive fields toward the attended location in an asymmetric manner (Connor et al., 1996; Maunsell and McAdams, 2000; Womelsdorf et al., 2008). Such asymmetric effects would be consistent with our findings that attending to the peripheral moving stimulus resulted in larger pRF sizes and more peripheral preferred pRF locations along the $x$-axis, compared with attending to the stationary central fixation point. In principle, these differences between attention conditions could be due to attention drawing pRFs toward the attended location in the attend stimulus and/or the attend fixation condition.

However, is it clear that, in total, our results cannot be explained by a general drawing of pRFs toward the attended location in the attend stimulus and/or attend fixation condition. First, we demonstrate hemispheric differences in parietal cortex, with more peripheral pRF locations in the attend stimulus relative to the attend fixation condition only in the left hemisphere. In the right hemisphere, there was no significant difference in overall pRF location between the two attention conditions. Second, the hemispheric differences in attentional modulation of horizontal pRF preferred location in IPS $0-5$ were not evident in the analysis of effects of attention on vertical pRF preferred location. Finally, pRF locations along the $y$-axis were not significantly affected by spatial attention in left and right IPS $0-5$, TO1/2, and V1-V3, despite clear attentional modulation of horizontal pRF preferred locations in these same areas during the same trials. Thus, a general drawing of pRFs toward the attended location (occurring either through shifts in pRF location or asymmetric reweighting of attenional gain) is inconsistent with our findings. However, the possibility of asymmetric effects of attention on pRF shape represents an important area for future research using model-free back projections of fMRI responses (Lee et al., 2013; Greene et al., 2014).

Our results are consistent with the greater incidence of hemispatial neglect after right compared with left hemisphere damage. Two different accounts have been proposed to explain this hemispheric asymmetry. Representational theories have suggested that right parietal cortex has maps that comprise the entire visual field (Mesulam, 1981; Pouget and Driver, 2000). On the other hand, the interhemispheric competition theory suggests that, in addition to directing attention to the contralateral visual field, each hemisphere also exerts inhibitory control over the opposite hemisphere (Kinsbourne, 1977; Cohen et al., 1994). Our results are consistent with elements of both of these theories. In support of representational models, we found that directing attention to the stimulus resulted in a more bilateral visual field representation in right but not left parietal cortex. Our results are also consistent with an interhemispheric account in which right parietal cortex exerts greater inhibition over left parietal cortex, suppressing ipsilateral visual field responses and thereby causing a predominantly contralateral representation in the left hemisphere. Although our study cannot definitively distinguish between these theories, it offers a method for further investigation of representational and interhemispheric competition accounts of hemispheric asymmetries that can be extended to other topographically organized areas, such as those in frontal cortex (Hagler and Sereno, 2006; Kastner et al., 2007).

A recent report found that, although attention increased pRF size of individual parietal voxels, it did not change the overall size of the visual field representation in individual topographically organized areas (Sprague and Serences, 2013). Rather, at the level of individual brain areas, attention increased response amplitude (attentional gain). The asymmetries we report here are consistent with attentional gain changes that are restricted to contralateral hemifield visual field locations in left IPS but present for both hemifields in right IPS. Our finding of greater visual field representation in right IPS in the attend stimulus relative to attend fixation condition may also be due to the fact that we separately analyzed left and right IPS and that our IPS region included IPS0 -5, whereas in Sprague and Serences (2013), the bilateral IPS region primarily corresponded to IPS0/1.

Perceptual load has also been shown to modulate pRF size and location in human topographic cortical areas (de Haas et al., 2014). Specifically, greater demands on attention during performance of a central fixation task were associated with larger $\mathrm{pRF}$ size in peripheral visual field representations and a greater eccentricity of pRF locations in early visual cortex, compared with a fixation task that was less difficult (de Haas et al., 2014). Interestingly, pRF locations showed the opposite pattern in topographic IPS0/1: more central locations when attention demands at fixation were greater. One notable difference between the two studies is that we equated task difficulty across attention conditions and varied the locus of spatial attention, whereas the task used by de Haas et al. (2014) required subjects to always maintain attention at central fixation while perceptual load was varied.

Intriguingly, behavioral effects associated with hemispheric asymmetries have been reported in neurotypical individuals (Posner et al., 1980; Asanowicz et al., 2012; Sheremata and Shomstein, 2014), and asymmetries in behavior correlate with individual differences in the degree of lateralization across hemispheres (Thiebaut de Schotten et al., 2011; Szczepanski and Kastner, 2013). However, consistent with behavioral results in previous fMRI studies reporting hemispheric asymmetries in contralateral bias of responses in topographic parietal cortex during attention (Szczepanski et al., 2010; Szczepanski and Kastner, 2013) and visual short-term memory (Sheremata et al., 2010) tasks, we did not find a significant difference in behavioral performance between the two visual hemifields. One possibility is that our behavioral measure in the scanner may not have been sufficiently sensitive to detect visual field asymmetries. Consistent with this possibility, we recently demonstrated load-dependent differences in visual short-term memory performance between the left and right visual fields (Sheremata and Shomstein, 2014). These differences were not observed behaviorally in our fMRI study on visual short-term memory, but they are consistent with the taskdependent hemispheric asymmetries in parietal fMRI responses observed in that study (Sheremata et al., 2010). Alternatively, performance in the motion direction discrimination task used in the present study may reflect activity in areas that preferentially process visual motion, such as TO1/2, that did not exhibit hemispheric asymmetries in the effects of attention on lateralization of visual field representations. Further investigation of the relationships between asymmetries in behavior and brain activity will be important for understanding hemispheric asymmetries in hemispatial neglect.

\section{References}

Amano K, Wandell BA, Dumoulin SO (2009) Visual field maps, population receptive field sizes, and visual field coverage in the human $\mathrm{MT}^{+}$complex. J Neurophysiol 102:2704-2718. CrossRef Medline

Asanowicz D, Marzecová A, Jaśkowski P, Wolski P (2012) Hemispheric asymmetry in the efficiency of attentional networks. Brain Cogn 79:117128. CrossRef Medline

Ben Hamed S, Duhamel JR, Bremmer F, Graf W (2002) Visual receptive field modulation in the lateral intraparietal area during attentive fixation and free gaze. Cereb Cortex 12:234-245. CrossRef Medline 
Bressler DW, Silver MA (2010) Spatial attention improves reliability of fMRI retinotopic mapping signals in occipital and parietal cortex. Neuroimage 53:526-533. CrossRef Medline

Bressler DW, Fortenbaugh FC, Robertson LC, Silver MA (2013) Visual spatial attention enhances the amplitude of positive and negative fMRI responses to visual stimulation in an eccentricity-dependent manner. Vision Res 85:104-112. CrossRef Medline

Cohen JD, Romero RD, Servan-Schreiber D, Farah MJ (1994) Mechanisms of spatial attention: the relation of macrostructure to microstructure in parietal neglect. J Cogn Neurosci 6:377-387. CrossRef Medline

Connor CE, Gallant JL, Preddie DC, Van Essen DC (1996) Responses in area $\mathrm{V} 4$ depend on the spatial relationship between stimulus and attention. J Neurophysiol 75:1306-1308. Medline

de Haas B, Schwarzkopf DS, Anderson EJ, Rees G (2014) Perceptual load affects spatial tuning of neuronal populations in human early visual cortex. Curr Biol 24:R66-R67. CrossRef Medline

Dumoulin SO, Wandell BA (2008) Population receptive field estimates in human visual cortex. Neuroimage 39:647-660. CrossRef Medline

Greene CA, Dumoulin SO, Harvey BM, Ress D (2014) Measurement of population receptive fields in human early visual cortex using backprojection tomography. J Vis 14:1-17. CrossRef Medline

Hagler DJ Jr, Sereno MI (2006) Spatial maps in frontal and prefrontal cortex. Neuroimage 29:567-577. CrossRef Medline

Hutchinson JB, Uncapher MR, Weiner KS, Bressler DW, Silver MA, Preston AR, Wagner AD (2014) Functional heterogeneity in posterior parietal cortex across attention and episodic memory retrieval. Cereb Cortex 24: 49-66. CrossRef Medline

Jenkinson M, Bannister P, Brady M, Smith S (2002) Improved optimization for the robust and accurate linear registration and motion correction of brain images. Neuroimage 17:825-841. CrossRef Medline

Kastner S, DeSimone K, Konen CS, Szczepanski SM, Weiner KS, Schneider KA (2007) Topographic maps in human frontal cortex revealed in memory-guided saccade and spatial working-memory tasks. J Neurophysiol 97:3494-3507. CrossRef Medline

Kinsbourne M (1977) Hemi-neglect and hemisphere rivalry. Adv Neurol 18:41-49. Medline

Lee S, Papanikolaou A, Logothetis NK, Smirnakis SM, Keliris GA (2013) A new method for estimating population receptive field topography in visual cortex. Neuroimage 81:144-157. CrossRef Medline

Maunsell JH, Van Essen DC (1987) Topographic organization of the middle temporal visual area in the macaque monkey: representational biases and the relationship to callosal connections and myeloarchitectonic boundaries. J Comp Neurol 266:535-555. CrossRef Medline

Maunsell JHR, McAdams CJ (2000) Effects of attention on neuronal response properties in visual cerebral cortex. In: The new cognitive neurosciences (Gazzaniga MS, ed), pp 315-324. Cambridge, MA: Massachusetts Institute of Technology.

Mesulam MM (1981) A cortical network for directed attention and unilateral neglect. Ann Neurol 10:309-325. CrossRef Medline

Niebergall R, Khayat PS, Treue S, Martinez-Trujillo JC (2011) Expansion of MT neurons' excitatory receptive fields during covert attentive tracking. J Neurosci 31:15499-15510. CrossRef Medline

Peirce JW (2009) Generating stimuli for neuroscience using PsychoPy. Front Neuroinform 2:10. CrossRef Medline

Posner MI, Snyder CR, Davidson BJ (1980) Attention and the detection of signals. J Exp Psychol 109:160-174. CrossRef Medline

Pouget A, Driver J (2000) Relating unilateral neglect to the neural coding of space. Curr Opin Neurobiol 10:242-249. CrossRef Medline
Ramachandran VS, Anstis SM (1990) Illusory displacement of equiluminous kinetic edges. Perception 19:611-616. CrossRef Medline

Roberts M, Delicato LS, Herrero J, Gieselmann MA, Thiele A (2007) Attention alters spatial integration in macaque $\mathrm{V} 1$ in an eccentricity-dependent manner. Nat Neurosci 10:1483-1491. CrossRef Medline

Sayres R, Grill-Spector K (2008) Relating retinotopic and object-selective responses in human lateral occipital cortex. J Neurophysiol 100:249-267. CrossRef Medline

Schluppeck D, Curtis CE, Glimcher PW, Heeger DJ (2006) Sustained activity in topographic areas of human posterior parietal cortex during memory-guided saccades. J Neurosci 26:5098-5108. CrossRef Medline

Sereno MI, Pitzalis S, Martinez A (2001) Mapping of contralateral space in retinotopic coordinates by a parietal cortical area in humans. Science 294:1350-1354. CrossRef Medline

Sheremata S, Shomstein S (2014) Hemifield asymmetries differentiate VSTM for single- and multiple-feature objects. Atten Percept Psychophys 76:1609-1619. CrossRef Medline

Sheremata SL, Bettencourt KC, Somers DC (2010) Hemispheric asymmetry in visuotopic posterior parietal cortex emerges with visual short-term memory load. J Neurosci 30:12581-12588. CrossRef Medline

Silver MA, Kastner S (2009) Topographic maps in human frontal and parietal cortex. Trends Cogn Sci 13:488-495. CrossRef Medline

Silver MA, Ress D, Heeger DJ (2005) Topographic maps of visual spatial attention in human parietal cortex. J Neurophysiol 94:1358-1371. CrossRef Medline

Sprague TC, Serences JT (2013) Attention modulates spatial priority maps in the human occipital, parietal and frontal cortices. Nat Neurosci 16: 1879-1887. CrossRef Medline

Swisher JD, Halko MA, Merabet LB, McMains SA, Somers DC (2007) Visual topography of human intraparietal sulcus. J Neurosci 27:5326-5337. CrossRef Medline

Szczepanski SM, Kastner S (2013) Shifting attentional priorities: control of spatial attention through hemispheric competition. J Neurosci 33:54115421. CrossRef Medline

Szczepanski SM, Konen CS, Kastner S (2010) Mechanisms of spatial attention control in frontal and parietal cortex. J Neurosci 30:148-160. CrossRef Medline

Thiebaut de Schotten M, Dell'Acqua F, Forkel SJ, Simmons A, Vergani F, Murphy DG, Catani M (2011) A lateralized brain network for visuospatial attention. Nat Neurosci 14:1245-1246. CrossRef Medline

Tootell RB, Hadjikhani N, Hall EK, Marrett S, Vanduffel W, Vaughan JT, Dale AM (1998) The retinotopy of visual spatial attention. Neuron 6:1409-1422. CrossRef Medline

Treue S (2001) Neural correlates of attention in primate visual cortex. Trends Neurosci 24:295-300. CrossRef Medline

Van Essen DC, Newsome WT, Maunsell JH (1984) The visual field representation in striate cortex of the macaque monkey: asymmetries, anisotropies, and individual variability. Vision Res 24:429-448. CrossRef Medline

Whitney D, Goltz HC, Thomas CG, Gati JS, Menon RS, Goodale MA (2003) Flexible retinotopy: motion-dependent position coding in the visual cortex. Science 302:878-881. CrossRef Medline

Womelsdorf T, Anton-Erxleben K, Pieper F, Treue S (2006) Dynamic shifts of visual receptive fields in cortical area MT by spatial attention. Nat Neurosci 9:1156-1160. CrossRef Medline

Womelsdorf T, Anton-Erxleben K, Treue S (2008) Receptive field shift and shrinkage in macaque middle temporal area through attentional gain modulation. J Neurosci 28:8934-8944. CrossRef Medline 